\title{
Controlling microbial co-culture based on substrate pulsing can lead to stability through differential fitness advantages
}

\author{
J.Andres Martinez ${ }^{1}$, Matheo Delvenne ${ }^{1, \boldsymbol{\bullet}}$, Lucas Henrion ${ }^{1, \boldsymbol{\bullet}}$, Fabian Moreno ${ }^{1, \boldsymbol{\bullet}}$, \\ Samuel Telek ${ }^{1}$, Christian Dusny ${ }^{2}$, Frank Delvigne ${ }^{1,{ }^{*}}$ \\ 1 TERRA Research and Teaching Centre, Microbial Processes and Interactions \\ (MiPI), Gembloux Agro-Bio Tech, University of Liége, Gembloux, Belgium. \\ 2 Microbial Single Cell Analysis, Department of Solar Materials, Helmholtz-Centre for \\ Environmental Research- UFZ Leipzig, Permoserstr. 1504318 Leipzig, Germany. \\ (2) These authors contributed equally to this work. \\ * f.delvigne@uliege.be
}

\begin{abstract}
Microbial consortia are exciting platforms for the bioproduction of complex metabolic products. However, the functional properties of microbial communities remain challenging to control, given the complex interactions between the co-cultured organisms. Microbial communities are invariably heterogeneous, possessing different phenotypic states compartmentalised in each microorganism. Furthermore, each strain can switch to alternative phenotypic states exhibiting different metabolic and fitness potentials. These transitions are related to the biological behaviour exhibited by cellular systems, leading to phenotypic diversification and fitness evolution processes. In this work, Escherichia coli and Saccharomyces cerevisiae were co-cultured with different feeding profiles designed to generate transitory environmental conditions and metabolic shifts, leading to the co-existence of the two microbial strains in continuous cultures. Intermittent feeding profiles allowed to generate temporal niches, providing fitness advantages to each strain, further ensuring co-culture stability. Single-strain cultures were used for inferring the growth and metabolic parameters for each strain. These parameters were then used to design a simplified cybernetic model for the co-culture, which simulated the consortium's performance under continuous and intermittent feeding profiles at various frequencies, feed step times and dilution rates. Two discontinuous feeding profiles were selected for co-culture experiments. Models and experiments pointed out that the intermittent process conditions allowed to produce alternating periodic conditions promoting the growth of E. coli and S. cerevisiae, enabling temporal niche fitness advantages for both strains. E. coli response was found to be less prone to substrate co-utilisation due to its greater catabolic repression features, while $S$. cerevisiae exhibited more flexibility regarding simultaneous carbon source utilisation. Experiments pointed out that the given intermittent feeding profiles could dynamically stabilise the co-existence of the two strains during long-lasting continuous cultivations. Furthermore, these specific frequencies and feeding profiles affected cellular interaction and community composition.
\end{abstract}

\section{Keywords:}

Synthetic co-culture, population control, phenotypic heterogeneity, stochastic modelling, environmental perturbation, continuous culture, bioengineering. 


\section{Introduction}

Microbial communities can be found in every habitat around all ecosystems, proving that within multiple organism compositions, it may be possible to gain access to an almost unlimited transformation capability 11. Microbial consortia, either natural or synthetic, have therefore been deemed as an exciting platform for bioproduction of new and ever more metabolites [1-3]. To date, microbial communities have been successfully used for many relevant industrial purposes, such as increasing crop productivity, bioremediation of soils and water resources, and the manufacturing of food and pharmaceutical products 1. 4]. Despite these achievements, the dynamic properties of cell population and the functional properties of microbial communities remain challenging to control and engineer, mainly because of the network of interactions between consortium organisms [1] Therefore, there has been an increased interest in studying strategies to control microbial consortia efficiently. In this regard, engineered microbial communities are desirable since, in theory, they would be capable of performing new transformation routes while providing less interaction complexity compared to naturally occurring consortia.

Population stability is one of the essential parameters to control in synthetic communities cultures. This control has been achieved with mitigated results, based on strategies primarily relying on the limitation of extracellular factors (such as substrate) and by genetic modifications. However, these strategies are built with limited knowledge about population dynamics and usually lead to reduced fitness or productivity 13 . Up to date, many different strategies have been studied to achieve the desired population stability, such as the use of specific carbon sources for each member of the community, the establishment of metabolic dependencies for growth of some strains, the insertion of genetic circuits for Spatio-temporal control over different cell functions, predator-prey systems or mutualism, and the compartmentalisation of metabolic tasks by biofilms or specific cultivation devices, along with different substrate and activator feeding control strategies $\left.3 \mid \begin{array}{lll}3 & 5 & 9\end{array}\right]$. In this context, the development of mathematical and computational frameworks capable of addressing the population dynamics of the co-cultured strains with an approach towards systemic complexity is of great interest for bioprocess engineering [8, 10].

Several mathematical and computational frameworks have been presented, addressing specific aspects linked with the complexity of the system, such as the multiplicity of cellular states 11], the stochasticity of intracellular reactions [12, the information conservation and its transmission across the different metabolic and cellular layers 12, 13, the cell size distributions and its impact on cell division rate 14]. The present work was aimed to contribute to understanding the systemic structure of microbial communities and their control. To this end, a modular computational framework incorporating phenotypic cell switching related to metabolic profile changes was constructed. The fitness advantage related to these phenotypic/metabolic switches will also be taken into account using cybernetic variables [15]. This modular computational framework was used to analyse the solution space for three different culture feed operation profiles (batch, continuous, and intermittent) in $E$. coli and $S$. cerevisiae co-cultures as a case study. The resulting responses in the solution space gave insight into plausible systemic characteristics of the process, such as elasticity and stability towards the control of the synthetic communities, providing helpful information for designing operational strategies to ensure population stability. 


\section{Materials and Methods}

\section{Mathematical and computational framework construction}

A simplified cybernetic mathematical framework for a single strain comprising different metabolite consumption and production states was constructed. Each single-strain cybernetic model was then used as a kernel section of a co-culture population computational framework (Fig. 1, A). A Monod base model was used for the individual strain rate equations. For this construction, let us first establish a common biochemical reaction for any substrate $S$ that can be consumed by the biomass $X$ at a given metabolic state as:

$$
X+Y_{\frac{s}{x}} S \stackrel{\mu_{\sigma}}{\longrightarrow} X+Y_{\frac{x}{x}} X+Y_{\frac{a}{x}} A+\ldots+Y_{\frac{n}{x}} N
$$

The latter biochemical reaction model allows to construct a stoichiometric vector $\phi$ for $S$ consumption such that the rate of change for any component in the media $M_{i}$ (including $X$ and $S, i=X, S, A, \ldots, N$ ) can be defined by the product of the growth rate $\mu_{\sigma}$ and $\phi$ vector as follows:

$$
\frac{d M}{d t}=\phi \mu_{\sigma}=\left[\begin{array}{c}
Y_{\frac{\sigma}{x}}^{\sigma} \\
Y_{\frac{\sigma}{x}}^{\sigma} \\
Y_{\frac{\sigma}{x}}^{\sigma} \\
\ldots \\
Y_{\frac{n}{x}}^{\sigma}
\end{array}\right] \mu_{\sigma}
$$

With this in mind, the model can then be extended to a stoichiometric Matrix where we can define each column as different $\phi$ for each known growth rate derived from the consumption of different substrates, which in turn can be associated with a specific metabolic state(e.g. the consumption of $A, \ldots, N)$. This extension then contains the known behavioural capabilities of the cell within the model $\Phi=\left[\phi_{s}, \phi_{a}, \ldots, \phi_{n}\right]$, such that:

$$
\frac{d M}{d t}=\Phi \vec{\mu}=\left[\begin{array}{cccc}
Y_{\frac{\alpha}{x}}^{\sigma} & Y_{\frac{\alpha}{x}}^{\alpha} & \ldots & Y_{\frac{\nu}{x}}^{\nu} \\
Y_{\frac{\sigma}{x}}^{\sigma} & Y_{\frac{s}{x}}^{\alpha} & \ldots & Y_{\frac{s}{x}}^{\nu} \\
Y_{\frac{\alpha}{x}}^{\sigma} & Y_{\frac{\alpha}{x}}^{\alpha} & \ldots & Y_{\frac{\alpha}{x}}^{\nu} \\
\ldots & \ldots & \ldots & \ldots \\
Y_{\frac{n}{x}}^{\sigma} & Y_{\frac{n}{x}}^{\alpha} & \ldots & Y_{\frac{n}{x}}^{\nu}
\end{array}\right]\left[\begin{array}{c}
\mu_{\sigma} \\
\mu_{\alpha} \\
\ldots \\
\mu_{\nu}
\end{array}\right]
$$

Each $\phi$ vector also contains the information for its effect on all components in the system. If a particular metabolite is not produced or consumed in a particular metabolic state, then its yield $(Y)$ is set to 0 . For our particular purpose, we will set that for a metabolic state for single primary substrate $(j \ldots j+n)$ consumption, the growth rate $\mu_{j \ldots j+n}$ is given by a simplified Monod-type equation such as:

$$
\mu_{j}=\frac{\mu_{j}^{\max } M_{j}}{K_{j}+M_{j}} X
$$

We can then rewrite Eq. 3 in a general form as follows:

$$
\frac{d M_{i}}{d t}=Y_{\frac{i}{x}}^{\sigma} \frac{\mu_{\sigma}^{\max } M_{\sigma}}{K_{\sigma}+M_{\sigma}} X+Y_{\frac{i}{x}}^{\alpha} \frac{\mu_{\alpha}^{\max } M_{\alpha}}{K_{\alpha}+M_{\alpha}} X+\ldots+Y_{\frac{i}{x}}^{\nu} \frac{\mu_{\nu}^{\max } M_{\nu}}{K_{\nu}+M_{\nu}} X
$$

This general form can then be reduced for each metabolite as some yields are 0 for a particular Substrate $j$. In this work, we used this equation as the base for the construction of the mathematical model to describe the growth, consumption and production behaviour of E.coli and S.cerevisiae strains. The conceptual model is presented in Fig. 4. In this 
figure, we can observe that the biomass $X$ can interact by consuming and producing three different external metabolites, Glucose (GLC, $G$ ), Acetate (ACE, $A$ ) and Ethanol (ETH, $E)$. These metabolites were chosen as they are the most relevant for the metabolism of both strains when cultured in minimal media with GLC as the only carbon source. The $\Phi$ matrix related equations constructed was then set to be the following:

$$
\frac{d M}{d t}=\Phi \vec{\mu}=\left[\begin{array}{cccc}
1 & 1 & 1 & 1 \\
Y_{\frac{g}{x}}^{\gamma o} & Y_{\frac{g}{x}}^{\gamma f} & 0 & 0 \\
0 & Y_{\frac{\alpha}{x}}^{\gamma f} & Y_{\frac{\alpha}{x}}^{\alpha} & 0 \\
0 & Y_{\frac{e}{x}}^{\gamma f} & 0 & Y_{\frac{\epsilon}{x}}^{\epsilon}
\end{array}\right]\left[\begin{array}{c}
\mu_{\gamma o} \\
\mu_{\gamma f} \\
\mu_{\alpha} \\
\mu_{\epsilon}
\end{array}\right]
$$

Where $\mu_{\gamma o} \mu_{\gamma f}$ are the oxidative, and fermentative growth on glucose, respectively, and $\mu_{\alpha}$ and $\mu_{\epsilon}$ are the growth rates during acetate and ethanol consumption. In this work, the growth rates were also set to be affected by a general metabolism inhibition proportioned by the external accumulation of the substrates as follows:

$$
\mu_{j}=\frac{\mu_{j}^{\max } M_{j}}{K_{j}+M_{j}} X H^{-}
$$

where:

$$
H^{-}=\Pi_{i}^{n} \frac{1}{1+\frac{M_{i}}{I_{i}}}
$$

Finally with the addition of a first order death rate, the equations used in the present work can be expressed as follows:

$$
\begin{aligned}
\frac{d G}{d t} & =\left[Y_{\frac{g}{x}}^{\gamma o} \frac{u_{\gamma o}^{\max } G}{K_{\gamma o}+G}+Y_{\frac{g}{x}}^{\gamma f} \frac{u_{\gamma f}^{\max } G}{K_{\gamma f}+G}\right] X H^{-} \\
\frac{d A}{d t} & =\left[Y_{\frac{a}{x}}^{\gamma f} \frac{q_{\max }^{\gamma f} G}{K_{\gamma f}+G}+Y_{\frac{a}{x}}^{\alpha f} \frac{q_{\max }^{\alpha} A}{K_{\alpha}+A}\right] X H^{-} \\
\frac{d E}{d t} & =\left[Y_{\frac{e}{x}}^{\gamma f} \frac{q_{\max }^{\gamma f} G}{K_{\gamma f}+G}+Y_{\frac{e}{x}}^{\epsilon f} \frac{q_{\max }^{\epsilon} E}{K_{\epsilon}+E}\right] X H^{-} \\
\frac{d X}{d t} & \left.=\left[\frac{q_{\max }^{\gamma o} G}{K_{\gamma o}+G}+\frac{q_{\max }^{\gamma f} G}{K_{\gamma f}+G}+\frac{q_{\max }^{\alpha} A}{K_{\alpha}+A}+\frac{q_{\max }^{\epsilon} E}{K_{\epsilon}+E}\right] X H^{-}-K_{d} X\right)
\end{aligned}
$$

Each $\Phi$ Matrix and Kinetic parameters pairs, approximated for each microorganism, are understood in this work as its single-strain model. E.coli and S.cerevisiae models were constructed in this work, approximated from their experimental behaviour in axenic cultures. Their combination into a mixed matrix gives rise to a co-culture model (Fig 1. A), with every strain having its own $\Phi$ sets at their rates $\vec{r}_{i}$ as the first order of interaction between them.

\section{Mathematical description for the Cybernetic framework}

The cybernetic modelling approach was used to extend the characterisation of the behaviour of the single cultures of S.cerevisiae and E. coli during the batch cultures and continuous culturing processes as it can render the allocation of cell's resources on several metabolic options. The cybernetic variables represent the expression of the metabolic machinery related to a particular substrate metabolism, a so-called "representative enzyme". This representative enzyme encompasses all the actual enzymes, co-factors, and other resources necessary for the metabolic reactions regarding a particular substrate consumption and fate [15. The cybernetic approach has been previously used to 
address the diauxic behaviour in Kleibsiela oxytoca [16], and more recently to derive full dynamic models into the metabolic fluxes across several microorganisms $[17-19]$ and even mammalian cells 17,20. A full description of the cybernetic approach can be found in Ramkrishna et al. reports [15, 16, 18, 20]. In this work, it was first assumed that each phenotype could be simplified as the consumption of one or more substrates $\left(M_{s}\right)$ catalysed by a critical enzyme $\left(E_{s}\right)$ for the production of biomass $X$ and other products $\left(M_{p}\right)$ [16. $E_{s}$ represents the set of all enzymes governing the kinetics of this specific phenotype. $E_{s}$ synthesis is induced by the presence of the specific substrate or metabolite $M_{s}$. This simplified model can be written as:

$$
\begin{aligned}
& X+M_{s} \stackrel{\Psi_{s}}{\longrightarrow}\left(1+Y_{x / s}\right) X+Y_{p / s} M_{p}+\ldots \\
& X+M_{s} \stackrel{M_{s}}{\longrightarrow} X^{\prime}+\Psi_{s}
\end{aligned}
$$

Where $X^{\prime}$ represents the biomass excluding the critical enzyme $\Psi_{s}$. These two reactions can be described by commonly used kinetic equations such as the Michaelis-Menten model for enzymatic catalysis. In this work these kinetic equations are derived from the previously shown mass balance model:

$$
\begin{aligned}
\frac{d m_{s}}{d t} & =\frac{q_{s} \psi_{s} M_{s} X}{K_{s}+M_{s}} H^{-} \\
\frac{d \psi_{s}}{d t} & =\alpha_{s}^{c}+\frac{\alpha_{s}^{i} M_{s} X}{K_{s}^{\prime}+M_{s}} H^{-}-\beta_{s} \psi_{s}-\mu \psi_{s}
\end{aligned}
$$

where $\alpha_{s}^{c}$ and $\alpha_{s}^{i}$ are the production rate constants for the enzyme for its constitutive and inducible expression, respectively. While $\beta_{s}$ is the decay constant of the enzyme. These parameters have been previously approximated for various microorganisms and cell lines including E.coli and S.cerevisiae 15 16. $\psi_{s}$ is the specific concentration of the enzyme $\Psi_{s}$ such that $\psi_{s} X$ is the total concentration of this enzyme. $\alpha_{s}^{c}+\alpha_{s}^{i}$ gives the maximum synthesis rate for this enzyme. The cybernetic approach solves the difficulty of calculating $\psi_{i}$ by assuming that the maximum quantity of enzyme defines the maximum rate. Therefore:

$$
\begin{aligned}
q_{s}^{\max } & =q_{s} \psi_{s}^{\max } \\
\psi_{s}^{\max } & =\frac{\alpha_{s}^{c}+\alpha_{s}^{i}}{u^{\max }+\beta_{s}}
\end{aligned}
$$

the enzyme concentration value can be substituted by a relative enzyme value respective to the maximum enzyme concentration as:

$$
q_{g} \psi_{g}=q_{g}^{\max }\left[\frac{\psi_{g}}{\psi_{g}^{\max }}\right]
$$

Finally, the cybernetic modelling introduces the regulation of the inhibition/activation of enzyme expression and repression/induction of enzyme activity by the introduction of the variables $v$ and $\nu$ which regulate enzyme synthesis $\frac{d \psi_{g}}{d t}$ and activity $\frac{d m_{g}}{d t}$ along with the model.

$$
\begin{aligned}
& \frac{d \Psi_{g}}{d t}=v_{g} \frac{d \psi_{g}}{d t} \quad\left(0<v_{g}<1 ; \sum_{j=g}^{e} v_{j}=1\right) \\
& \frac{d M_{g}}{d t}=\nu_{g} \frac{d m_{g}}{d t} \quad\left(0 \leq \nu_{g} \leq 1\right)
\end{aligned}
$$


The cybernetic variables $v$ and $\nu$ are calculated by matching law equations constructed for specific metabolic objectives. In the case of this work, the growth rate was selected as the metabolic objective, which in turn represents a fitness index. Cybernetic variables can then be understood as the comparison between the fitness advantage return for each reaction driven by each $\Psi_{\iota \ldots \omega}$ and this can be used to regulate its participation in the cellular metabolism at any time. The equations used for these cybernetic variables are the following:

$$
\begin{aligned}
v_{\iota} & =\frac{\mu_{\iota}}{\sum_{j=\iota}^{\omega} \mu_{j}} \\
\nu_{\iota} & =\frac{\mu_{\iota}}{\max \left(\mu_{\iota \ldots \omega}\right)}
\end{aligned}
$$

where $\mu_{g}$ represents the growth rate supported by each reaction driven by each $\Psi_{\iota \ldots \omega}$ in this case by the consumption of glucose $\Psi_{g}$, acetate $\Psi_{a}$ and ethanol $\Psi_{e}$. In this way, a dynamic distribution of its participation can be calculated to describe the metabolic and physiological behavior $\Phi$ given a metabolic reaction network 15, 16, 18, 21]. The approach allows to approximate the current phenotype by describing the metabolite content $\left(M_{\iota \ldots \omega}\right)$, enzymatic content $\left(\Psi_{\iota \ldots \omega}\right)$ and its functional relationship given by the regulation $\left(v_{\iota \ldots \omega}\right.$ and $\left.\nu_{\iota \ldots \omega}\right)$.

$$
\Phi \supset\{\Psi \stackrel{v \nu}{\longrightarrow} M\}
$$

In this work, the cybernetic model was implemented in Matlab, and the models were fitted to experimental data evaluated with the Willmot index (WLM) 22 24.

\section{Simulation design and toolbox}

Co-culture models were then used to generate simulations for the population behaviour in continuous and discontinuous cultures. Three different parameters were taken into account, Dilution rate $(D)$, frequency of pulsing $(w)$, and pulse-step percentage $(s)$. For continuous processes, the pulsing was performed on the concentration of the primary substrate, in this case, GLC, whereas for intermittent processes, pulses were applied to the dilution rate. The intermittent feeding regimes in this research were determined by a classical square wave function where the step-time determines the symmetry of the pulse. The latter means that the parameter $s$ with a value of 50 pulses half the time between the start of the new cycle, while a value of 100 produces a continuous culture with no changes in the up (pulsed) operation state, and a 0 valued $s$ produces a continuous culture with no changes in the down (non-pulsed) operation state. Simulations were performed in a constructed framework that allows the numeric integration of the previously described Monod-type models with the environmental (fermentation) conditions. The codes were constructed in MATLAB as the Monod-type Co-culture Kinetic Simulation (MONCKS) toolbox. The example code is available at https: //gitlab.uliege.be/mipi/modelingframeworks/moncks, and a simplified simulation workflow can be viewed in Fig. 1 B.

\section{Strains and medium composition}

Strains used in this study are E.coli K-12 W3110 and S. cerevisiae CEN-PK 117D, which grow on minimal media. The strains were maintained at $-80{ }^{\circ} \mathrm{C}$ in working vials $(2 \mathrm{~mL})$ in LB with $30 \%$ glycerol $(\mathrm{w} / \mathrm{v})$. Precultures and cultures were performed on synthetic media according to Verduyn et al. 25], but with modified phosphate buffer 


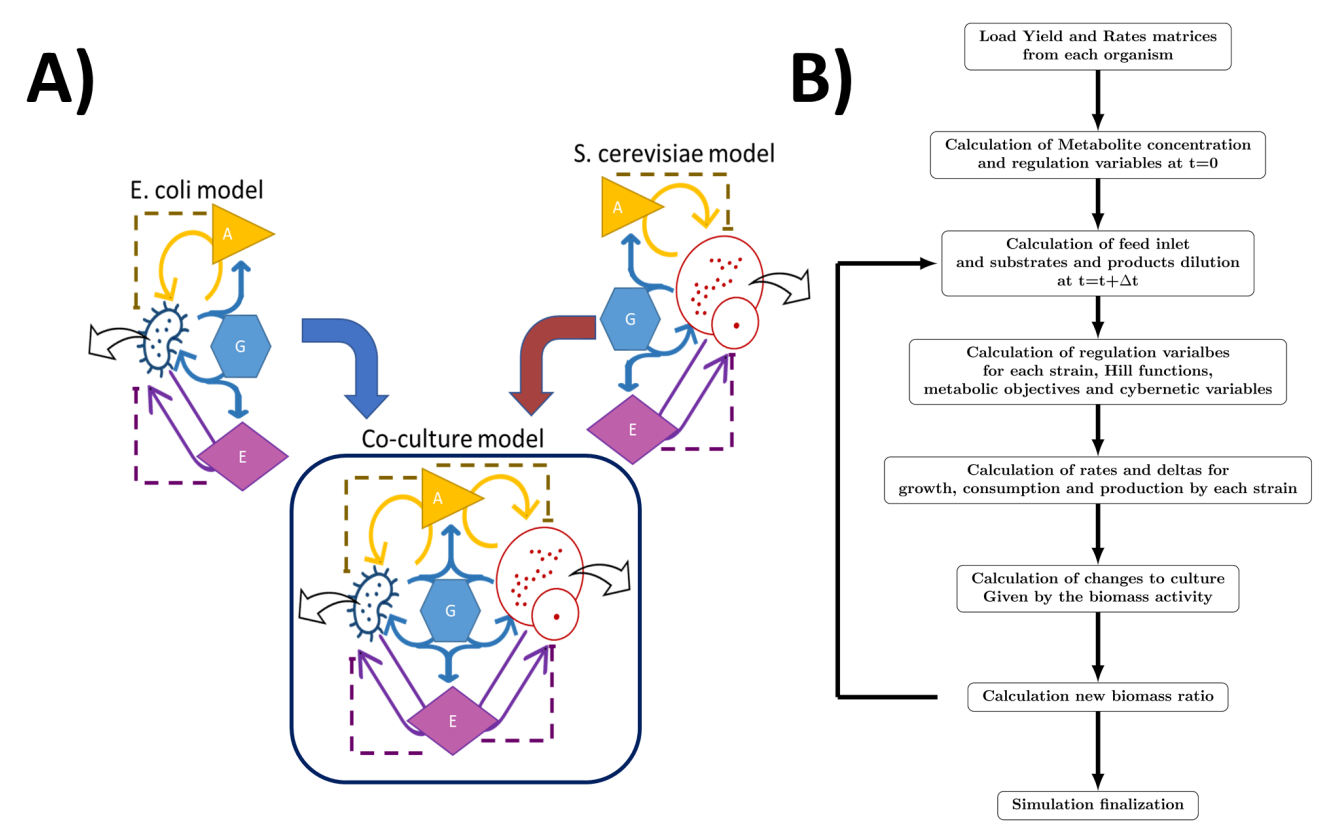

Fig 1. A) Simplified representation for the construction of the model for co-culture experiments. Each monoculture model is taken as a compartment on a virtual cell with separated metabolic objectives for cybernetic variable calculations. GLC in light blue, ACE in yellow, ETH in purple.S.cerevisiae and E.coli biomass are shown in red and in dark blue respectively. B)Simplified algorithm steps diagram used in MONCKS framework used for simulating the co-culture experiments

proportions of potassium dihydrogen phosphate $(6.309 \mathrm{~g} / \mathrm{L})$ and potassium hydrogen phosphate $(9.34 \mathrm{~g} / \mathrm{L})$ at $\mathrm{pH} 6.8$ and supplemented with variable glucose concentrations (Sigma-Aldrich, US). Biomass conversions from Biomass (g/L) to OD600 and number of cells were performed with data obtained form literature 26$] 28$.

\section{Culture procedures}

A micro-reactor experimental setup was used to derive $\mu, \mathrm{q}, \mathrm{Ks}$, and cell size distribution. A BioLector/RoboLector (M2PLabs, Germany) device was used to perform parallel fermentations in a $6 \times 8$ plate, in which wells would be stopped and sampled at different times. Every eight wells were set to be a single parallel fermentation and sequentially sampled every 3 hours until 24 hours. This work performed initial parameter characterisation experiments with $20 \mathrm{~g} / \mathrm{L}$ and $1.25 \mathrm{~g} / \mathrm{L}$ initial glucose concentration at $\mathrm{pH}$ 6.8. The initial biomass concentration was set to be approximately 0.1 OD. Time $0 \mathrm{~h}$ measurements were obtained by mixing outside wells the same amount of media and preculture and immediately processing it as a sample. The shaking frequency was set to 1000. Samples were separated into two vials, one was used for flow cytometry analysis, and the other was immediately filtered and prepared for glucose and organic acid measurement by HPLC.

The Co-culture experiments were performed with separated precultures grown for each strain and mixed at inoculation time. Population ratio of 1:1 (S.cerevisiae:E.coli in $\mathrm{g} / \mathrm{L}$ units) was used as the initial condition. Co-cultures in different fermentation regimes were done on lab-scale stirred bioreactors (Biostat B-Twin, Sartorius). The 
processes were performed at a $1 \mathrm{~L}$ working volume with a $1 \mathrm{~L}$ volume of headspace. The cultures were started for every experiment with a batch phase in modified Verduyn media, temperature maintained at $37^{\circ} \mathrm{C}, \mathrm{pH}$ at 6.8 , stirring rate of $1000 \mathrm{rpm}$, and aeration rate of 1 VVM. Upon 10 hours of cultivation, the feed was started. Three different feed regimes were used in continuous culture, at $0.1 \mathrm{~h}^{-1}$ dilution rate, one continuous and two intermittent operations with different combinations of frequency and time step derived from the simulations.

\section{Analytical sampling processing}

Samples taken from were processed for glucose and organic acid measurement by HPLC with an Aminex HPX-87H column(Bio-Rad, Hercules CA, USA) at $45{ }^{\circ} \mathrm{C}$, and $5 \mathrm{mM}$ sulfuric acid as mobile phase. An Agilent 1200 Series HPLC system was used with a refraction index detector at $50{ }^{\circ} \mathrm{C}$ (Agilent, Santa Clara CA, USA). Values below quantification limit were taken as zero for model fitting purposes. Cytometry data was obtained with an Accuri C6 flow cytometer (BD Accuri, San Jose CA, USA) 29. The sample was first tested on the $\mathrm{C} 6 \mathrm{FC}$ to measure the events/L and prepared by dilution until a concentration below 1000. Diluted samples are then fed into the $\mathrm{C} 6 \mathrm{FC}$ for analysis at an average flow rate of $14 \mu \mathrm{l} / \mathrm{min}$ with a threshold FSC-H set at 40000 . The analysis ended after collecting at least 40,000 events or $70 \mu \mathrm{L}$ of a sample.

\section{Results and Discussion}

\section{Cybernetic model reveal differences in metabolic profile when microbial strains are growing alone}

The individual characterisation of the strains was performed on minimal media, biomass, GLC, ACE, and ETH being followed through the process (Fig. 2). It can be observed that S.cerevisiae exhibits a slightly higher yield on these cultivation conditions than E.coli. The apparent yield computed from the approximation of maximum produced biomass and total GLC exhaustion for S.cerevisiae is $0.30 \pm 0.04$, whereas E.coli has a $0.23 \pm 0.07$. The observed global yield increase in S.cerevisiae may be the consequence of a more balanced glucose consumption metabolism at smaller concentrations and consumption rates [30]. We can observe a two-step growth curve in S.cerevisiae which may be related to its general preference for respiro-fermentative metabolism when growing at near maximum growth rates or under oxygen-limited conditions 31.

The global metabolic profiles obtained with the E.coli and S.cerevisiae models follow reasonably the experimental trends. E.coli presents sequential GLC and ACE utilization while S.cerevisiae ETH and ACE are co-utilised. The latter leads to longer second growth phase than the observed for E.coli. Even if E.coli can produce ETH through an alcohol dehydrogenase, the strict aerobic conditions used in this work limit its production 32. Therefore, three different metabolic states were considered, two for GLC consumption, one for ACE consumption, and one extra for S.cerevisiae comprising ETH consumption. GLC consumption states were divided into fermentative/unbalanced and oxidative/balanced GLC consumptions. In general, S.cerevisiae had higher yields and smaller saturation constants while E.coli had higher growth and consumption rates (Table 1). The approximated parameters suggest that E.coli would outgrow S.cerevisiae in almost every condition. However, S.cerevisiae could benefit from a more heterogeneous metabolism during substrate limitation or transitory metabolite shifts. 

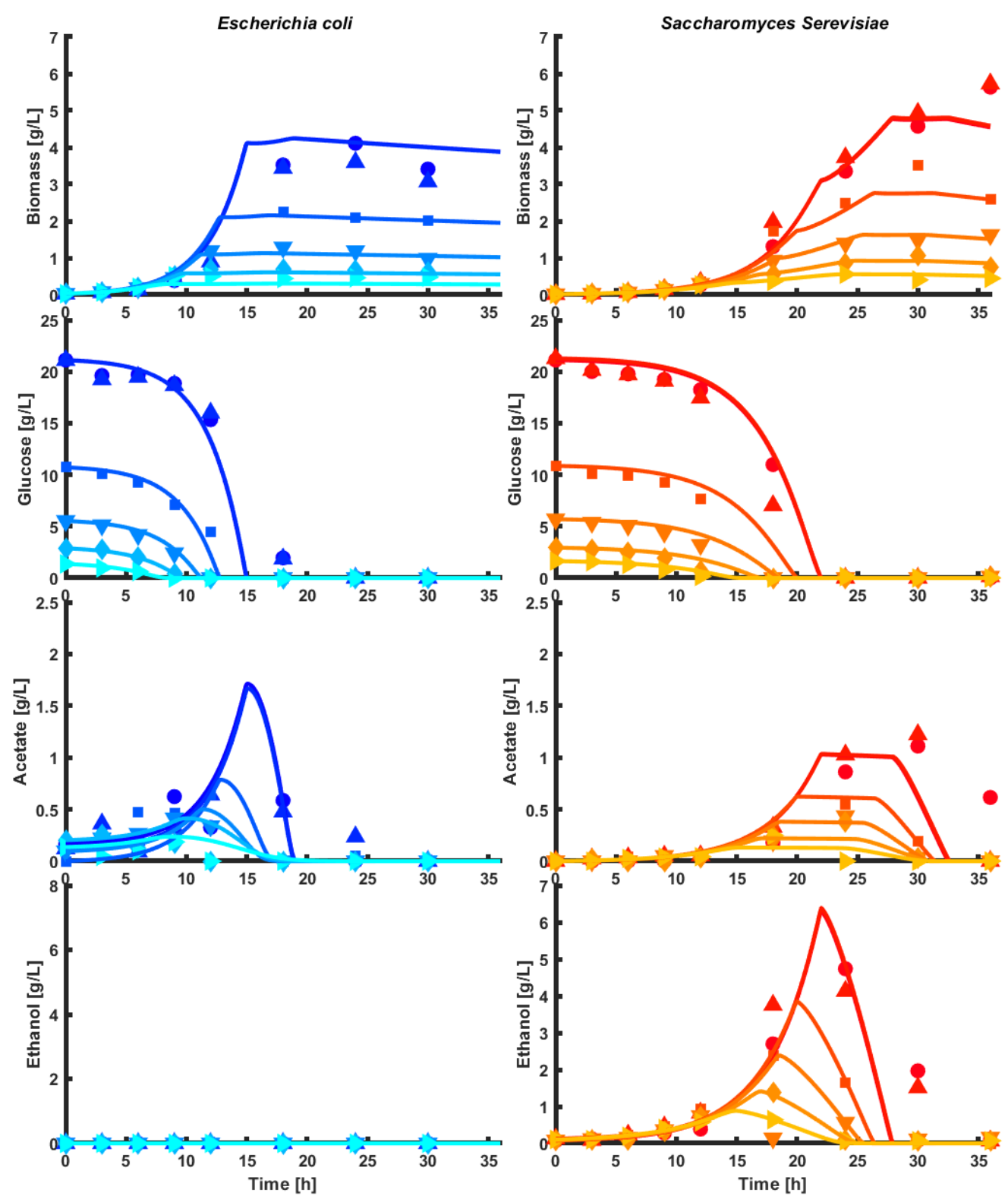

Fig 2. E.coli, S.cerevisiae biomass growth and metabolite profiles on microplate bioreactors at different starting glucose concentrations $\left([G L C]_{i}\right)$. Dark-blue to ligh-blue E.coli in 20 to $1.25 \mathrm{~g} / \mathrm{L}$ of initial GLC, dark-red to light-red S. cerevisiae in 20 to $1.25 \mathrm{~g} / \mathrm{L}$ of initial GLC

Metabolic heterogeneity is relevant for promoting the interactions needed for establishing microbial communities 33 34. Thattai and van Oudenaarden 11 established that cell-to-cell differences in gene expression and protein translation (enzymes concentration and activity) could split the population into two or more groups, each one presenting different phenotypic behaviour. Autocatalytic loops can further increase the existence of multistability in cell regulatory networks 11,12. The multiplicity of steady states in continuous cultures produces cells with different metabolic states [35]. Finally, cells can 
Table 1. Biomass maximum growth rates, saturation constants,maximum substrate consumption rates and biomass/substrate yields obtained from modeling approximation to monoculture fermentations.

\begin{tabular}{|l|cc|cc|cc|cc|}
\hline Substrate & \multicolumn{2}{|c|}{$u_{\max }$} & \multicolumn{2}{c|}{$K_{s}$} & \multicolumn{2}{c|}{$q_{\max }^{\text {s }}$} & \multicolumn{2}{c|}{$Y_{x / s}$} \\
\hline \multirow{3}{*}{ GLC(ferm) } & $E . c o l i$ & S.cer & E.coli & S.cer & E.coli & S.cer & E.coli & S.cer \\
\cline { 2 - 10 } ACE & 0.354 & 0.275 & 0.131 & 0.074 & -1.796 & -1.313 & 0.197 & 0.209 \\
ETH & 0.077 & 0.017 & 0.111 & 0.007 & -0.611 & -0.055 & 0.127 & 0.300 \\
GLC(Ox) & - & 0.104 & - & 0.049 & - & -0.331 & - & 0.315 \\
\hline
\end{tabular}

switch between the different states, and the rates of these transitions are functions of the environmental conditions 292936,37 . Therefore it becomes possible to vary the distribution of cells between various states as a function of the environment and time [1],34. Furthermore, while co-cultured, interactions between inter and intra-specific metabolic states may change the microorganism interactions and its population equilibrium and stability.

Models address this distribution of metabolic states with the use of cybernetic variables. In Fig. 3, the behaviour of the relative enzyme concentration for each metabolic state (substrate consumption and conversion) can be visualised. The relative enzyme values for the fermentative/unbalanced growth on GLC for E.coli start at their maximum value, and as long as the concentration remains non-limiting $(<1.5 \mathrm{~g} / \mathrm{L})$. Balanced growth vector has almost no participation derived from its relative enzyme and effective growth rate values (Fig. 3). After this GLC exhaustion threshold, the relative enzyme for ACE consumption increases and peaks ( 0.4 arbitrary units) at $\approx 17$ hours. However, even in this relative enzyme concentration has peaked, the contribution of ACE derived growth rate is low compared to GLC consumption. These observations and parameter values suggest co-utilisation in the present conditions cannot be expected.

S.cerevisiae metabolism starts similarly with high glucose consumption values, but the participation of the oxidative/balance growth, has higher growth participation values $(\approx 0.025-0.16)$ and increases as GLC initial concentration increases. Moreover, S.cerevisiae presents a weaker catabolite repression compared to E.coli. From the culture beginning, increases in the ETH catabolism are observed even before GLC exhaustion. Conversely to E.coli, the combination of growth rates and saturation constants provides softer catabolic repression allowing the temporary co-utilisation of multiple substrates. The relative enzyme profiles of S.cerevisiae show faster accumulation/decay dynamics, suggesting higher flexibility for the temporal allocation of metabolic resources. This characteristic indicates that $S$.cerevisiae could more easily present mixed metabolism in low concentrated mixed substrates, while E.coli would probably strongly commit to the fastest growing substrate. As can be observed, the information obtained by the cybernetic variables allows for the segregation of growth and fitness across different metabolic states, such as the consumption of different substrates [16].

\section{Fitness disparity between metabolic phenotypes leads to popula- tion imbalances during continuous cultures.}

The single strain models suggested significantly different resource allocation for each strain, given the relative enzyme production and degradation, especially when approaching substrate limitation conditions. The found differences derive from fitness differences 

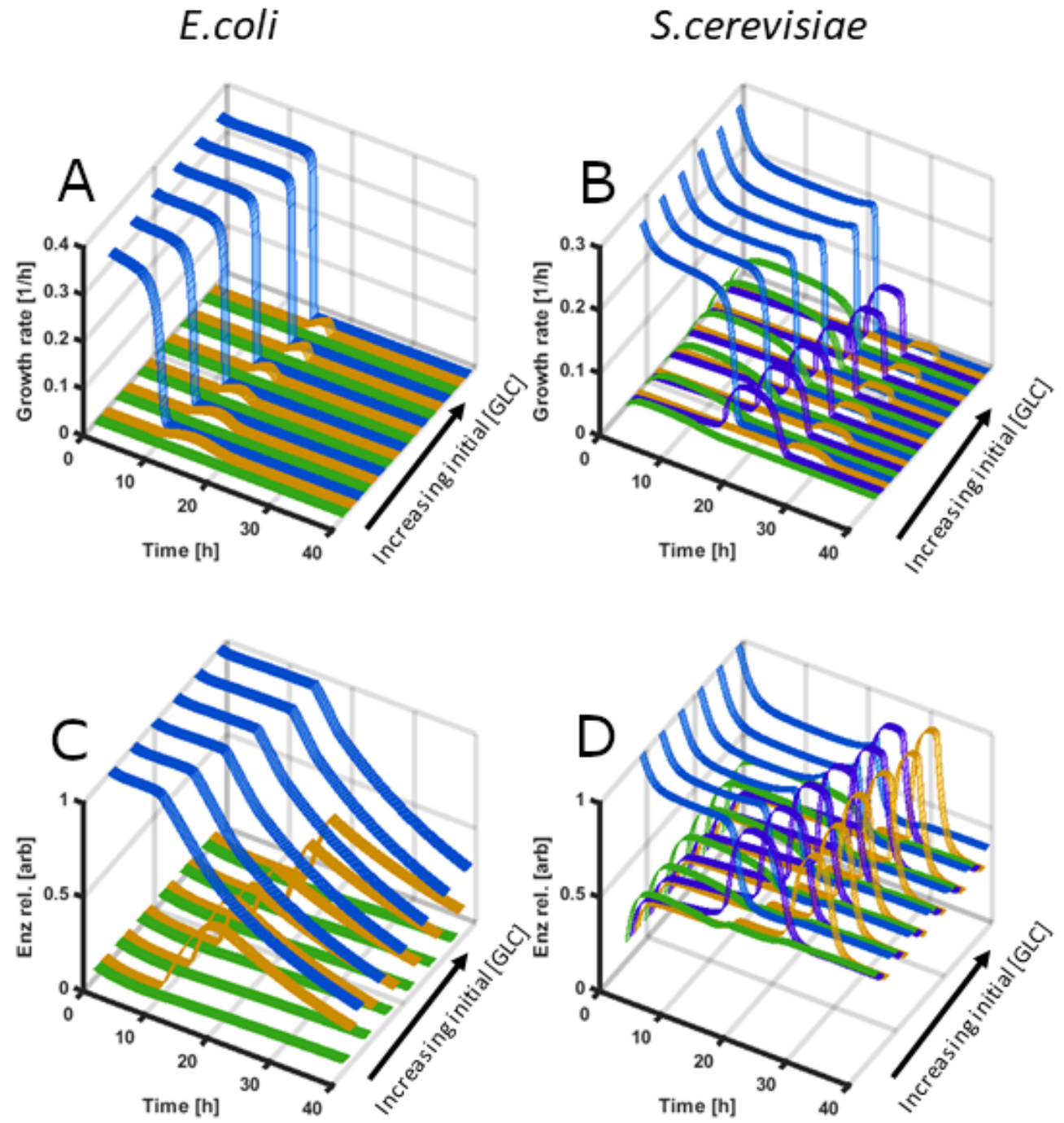

Fig 3. A and B Growth, rate contributions by each metabolic state for E.coli and S.cerevisiae, respectively. C and D, behaviour of the cybernetic variable of relative enzyme concentration for textitE.coli and S.cerevisiae, respectively. Metabolic states are color coded as follows: GLC fermentative/unbalanced consumption in light blue, ACE consumption in yellow, ETH consumption in purple and GLC oxidative/balanced consumption in green.

and their capability to switch between the diverse metabolic states. Balancing fitness across this metabolic heterogeneity is one of the main factors contributing to the microorganism population state and stability limits on a community system 10 .

In Fig. 4 the behavior of continuous co-cultures of the E.coli and S.cerevisiae strains with $0.1 h^{-1}$ as the Dilution feed with a $30 \mathrm{~g} / \mathrm{L}$ GLC Verduyn fresh media can be observed. Inhibition values between the strains were added to account for possible negative social interactions. However, similarly to the individual models, the inhibition values contributions on consumption and growth remained negligible compared to the changes in growth given by other parameters. Fig. 4 A presents the relative abundance of E.coli 
and S.cerevisiae, in comparison with the approximation of the biomass fraction obtained by FC (sum of the areas of the events corresponding to each strain). In this Fig., it can be observed a rapid decay of the population of S.cerevisiae during the initial sections of the culture when a GLC is at full availability. The consumption and growth rates dominate the population change during this first culture section. Given the disparity between the co-cultured strains, E.coli dominates the competition and the culture. However, after GLC enters limitation concentrations S.cerevisiae proportion increases during an $\approx 10$ hours, which is probably given by the consumption of initially the accumulated ETH, $\mathrm{ACE}$ and other internal carbon storage systems. After this time, its population starts to be slowly diminished in the system, having less than $3 \%$ of relative abundance by hour 80 .

Population stability is vital for the efficient use of these communities in an industrial framework 33, 38, 39. However, this is challenging to achieve when the phenotypical fitness differences between strains are significant, specifically in growth rate, substrate consumption rates, and substrate affinity / citeJawed2019. Furthermore, in a continuous feed operated reactor, which is stationary equilibrium driven, a narrow space for growth rate solutions is achieved, committing the system (community) to select only the best fitness solutions (highest growth individuals). Consequently, when co-culturing two strains with vastly different growth rates on single substrate addition in a continuous reactor, the slowest growing population (commonly the slowest for substrate intake) is washed out from the reactor chamber before arriving at a stationary state.

Fig. 4 B presents the metabolite concentrations during the fermentation simulation and its comparison to experimental data obtained in triplicate with their respective standard deviations. Fig. 4 B the experimentally observed optical density measurement (OD600) and the simulated total biomass on the green, with the individual microorganism biomass expected optical density. In continuous culture, substrate limiting conditions are expected. In Fig. 4 B, it is observed that after four retention times (or 40 hours), only marginal concentrations of GLC, ACE and ETH can be observed with values near the quantification limit. In these conditions, the E.coli, commitment to the GLC and its higher consumption and growth rates outcompete S.cerevisiae which would be consuming GLC but possibly also scavenging for other metabolic substrate traces in media, reducing its fitness in comparison to the former microorganism. Therefore, the washout of S.cerevisiae is due to the imbalanced differential fitness between the strains for sole GLC consumption. This imbalance could be solved by adding to the feed a substrate that S.cerevisiae prefers and E.coli is incapable of using or consumes with less efficiency, such as ETH, balancing the fitness and allowing a higher proportion of the former during the cultivation [?]. However, this is often more expensive or industrially unfeasible given the necessity of modifying the inlet influx and adding more unitary processes upstream. An alternative that can be devised to overcome this fitness imbalance is to counter it by allowing the system to temporarily enter concentration states into which S.cerevisiae would have better fitness balance towards E.coli by fluctuating the concentration profile of GLC in the bioreactor via either intermittent feeding. These fluctuating substrate conditions could then be tuned to the co-culture system and their microorganism transition metabolism characteristics to render different temporal fitness balances, impacting the microorganism population balance during the fermentation. 



Fig 4. Continuous co-culture simulations (dashed lines) and experiments (continuous lines or discrete points). A) Mass fraction for the two strains. Experimental data have been extracted from on-line flow cytometry S.cerevisiae in red and E.coli in dark blue. B) Metabolite profiles during the continuous cultivation. Metabolites in $\mathrm{g} / \mathrm{L}$, biomass in OD600. Total apparent biomass OD600 in green.

Fluctuating environmental conditions can introduce alternating fitness advantage windows between the co-cultured microorganisms.

The co-culture model was subjected to fermentation simulations where different feed profiles were set as input (Fig. 5p. The intermittent feeding profiles would produce different temporal substrates availabilities, which would produce shifts in the metabolic states of the different microorganisms. The rate of switching between these conditions and metabolic states would allow for temporal windows of favourable fitness advantage, allowing the system to enter dynamic population stability (Fig. 5).

The simulation results can be observed in Fig. 6 A, where each graph has the relative abundance of both microorganisms across time. It can be observed that as the dilution 


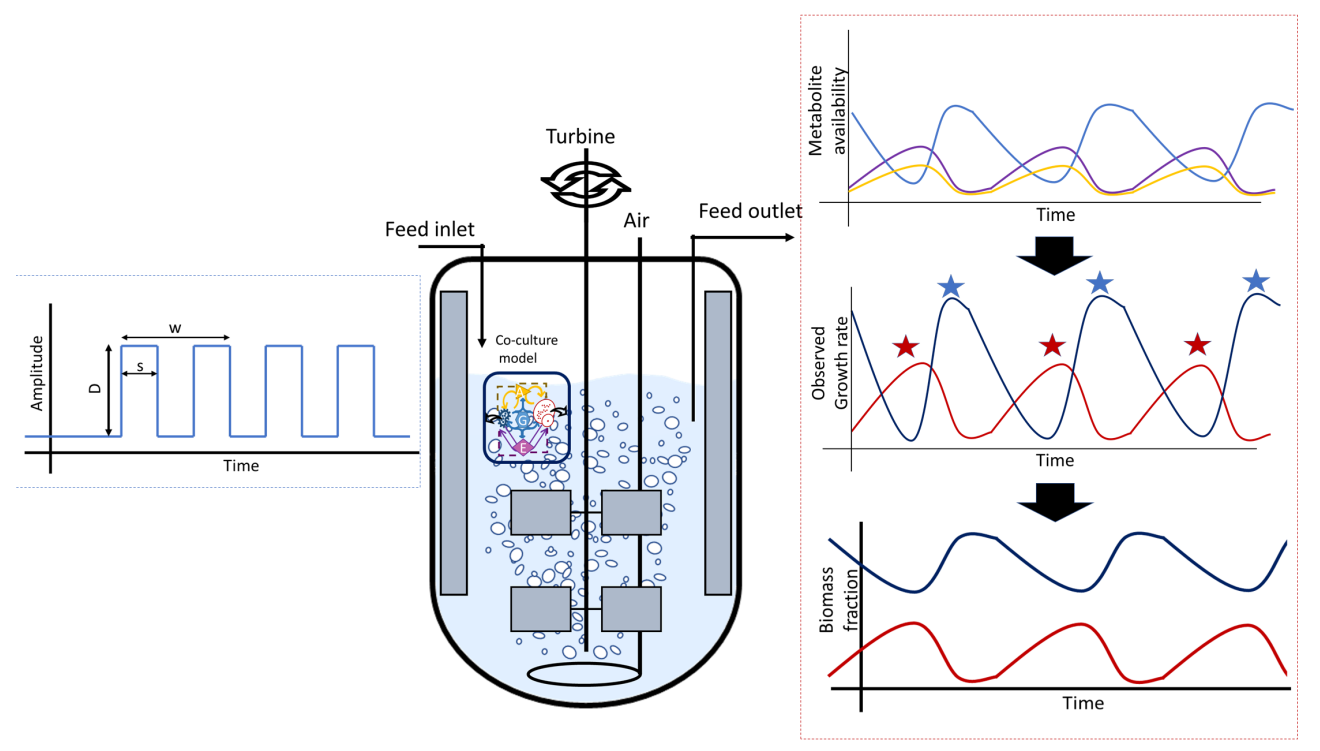

Fig 5. Simplified representation for the simulations design for various intermittent feeding profiles. The feeding profiles are considered as the system input and can be represented by square waves with dilution rate as the dependable variable. The simulated system comprises the co-culture of the two microbial strains and the environmental conditions i.e., the different metabolites and substrates concentrations . Model outputs are the instantaneous metabolite concentrations, observed growths and biomass fraction profiles. Stars mark the desired windows of preferential fitness for each strain. S.cerevisiae in red and E.coli in dark blue.

rate increases, the fractional allocation of E.coli increases more rapidly. For dilutions above 0.1 , several feed profiles exist that produce total S.cerevisiae washout. At dilutions closer to 0.3 , this washout occurs in time frames below $48 \mathrm{~h}$. In all dilution rates, the simulation couple around a value of 0.6 for E.coli and 0.4 for S.cerevisiae corresponds to sets comprising of no-feed operation. A gap between the latter and other feed simulations can be observed maximised at At the highest dilution rate tested $\left(0.3 h^{-1}\right)$. This gap reduces as the dilution rate decreases, probably due to a reduced growth ratio imbalance between strains.

Interestingly, in dilution rates of 0.1 and 0.025 , relative abundance profiles of some feed simulations cross these no-feed simulation lines, meaning that some feeding profiles could, if allowed to continue unchanged, derive in a S.cerevisiae extensive colonisation of the bioreactor, reversing the population balance upon long-term cultivation. The co-culture relative abundance between microorganisms seems to have a broad sensibility to intermittent feeding profiles. The latter suggests that the temporal displacement of metabolite concentrations could give rise to different fitness balances for the metabolic consumption options in the different strains. The impact of environmental fluctuations on the stability of microbial communities has been previously investigated and is known to affect community composition and functionality $8,40,42$. The search for stability conditions is a hot topic in the field of systems and synthetic ecology [43, 44 .

In Fig. 6 $\mathrm{B}$, the calculated instantaneous growth rate under a $0.1 h^{-1}$ dilution rate for all the metabolic states are presented for some intermittent feed profiles. One relevant observation is that during intermittence, faster growth rates for S.cerevisiae in comparison to E.coli can be observed. These temporary fitness advantages become more 
A)
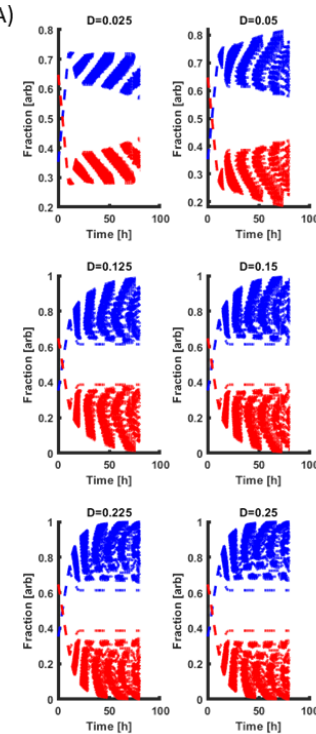
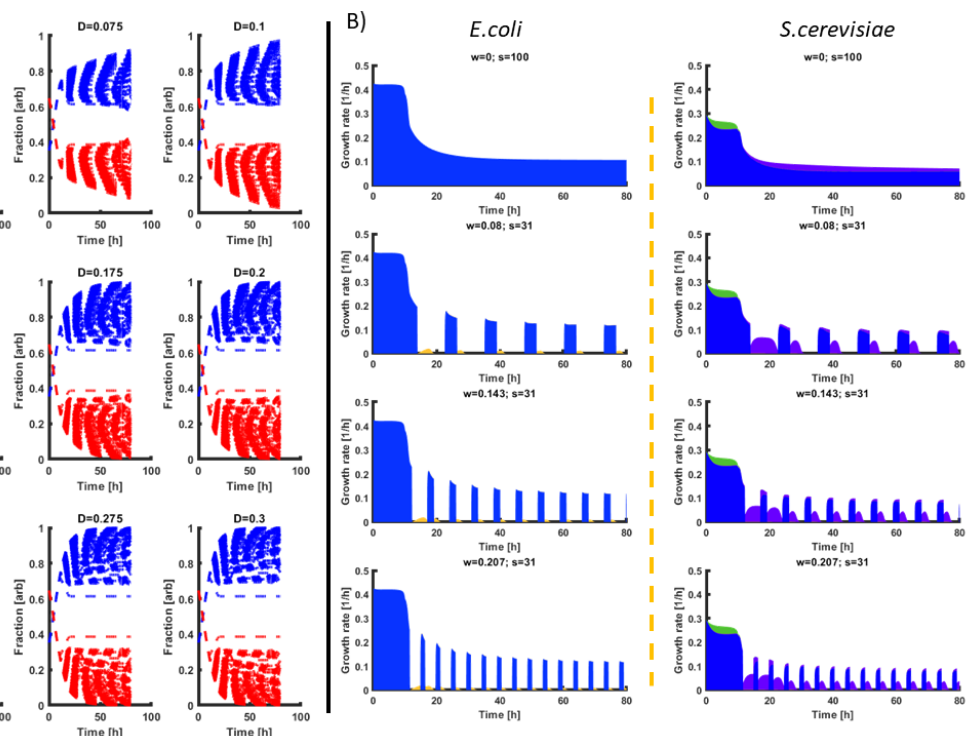

Fig 6. A)Fraction for each microorganism found for the semi-continuous fermentation simulations at different pulsating profiles. E.coli (red) and S.cerevisiae (blue). B) Accumulated instantaneous growth rate profiles for each strain for some simulations at different frequencies (w) and step-times(s). In this figure, growth supported from the different metabolic states are color coded as follows: GLC fermentative/unbalanced consumption in light blue, ACE consumption in yellow, ETH consumption in purple and GLC oxidative/balanced consumption in green.

common and pronounced as the frequency Increases and the step time reduces. The latter can be achieved because, in higher frequencies, more GLC would be input to the system allowing for more ETH production, while the lower step times mean that there is more time for S.cerevisiae to outgrow E.coli relying on the produced ACE. In Fig. 66B, it can be observed that a separation between growth rate contribution is found between the fermentative/imbalance GLC catabolic consumption and the ACE-dependent growth for E.coli. Conversely, the distribution of metabolic options for S.cerevisiae at any dilution rate and conditions seems to result in GLC and ETH co-utilisation. Therefore, E.coli has a better overall fitness in constant limiting GLC conditions (Fig. 66B), while S.cerevisiae can subsist in changing substrate environments where adaptation of the substrate uptake is needed. Furthermore, in the intermittent conditions (Fig. 6, B), the model predicts higher growth rates for S.cerevisiae when GLC is not available in the system due to better alternative carbon source utilisation (ETH). Therefore, S.cerevisiae can have a smaller mean fitness imbalance towards E.coli given by the ETH re-consumption metabolism in defined intermittent feeds.

The fact that cell populations can respond better to some stimulation frequencies has been previously explained from the perspective of natural frequencies of gene circuits [45], information transmission 46 or from the perspective of phenotypic switching and fitness advantage 10 11. The latter raises the possibility of exerting control into the population composition by adjusting the feed input, which will adjust the transitions between the metabolic possibilities available in each strain. 


\section{Intermittent feeding regimes can enhance population balance and stability during continuous co-culture fermentations.}

In order to challenge the above-mentioned hypothesis, two different feeding profiles were used for intermittent continuous co-culture experiments, the first at a lower frequency of $0.14 h^{-1}$ and a faster one with a frequency of $0.33 h^{-1}$ with steps percentages of $28.6 \%$ and $33.3 \%$, respectively. This combination of flux parameters results in square waves with 7 and 3 hours periods and "on" times of 2 and 1 hours, respectively. In Fig. ?? A, the flow cytometry plots for the times 10,48 , and $80 \mathrm{~h}$ can be found for one replicate of the continuous and the hi-freq and low-freq intermittent co-culture experiments. Conversely to the continuous operation, the observed density for S.cerevisae in both intermittent feed experiments seems to increase through the fermentation with an apparent net gain at $80 \mathrm{~h}$. Interestingly, the E.coli cluster centroid remains within the same range of Front Scattering Area (FSC-A) values for all experiments. This stability suggests that the events' mean size (cells) is maintained. However, it is also observed that a tail of increasing FSC-A values events is enriched for the intermittent experiments, being more represented on the low-frequency experiments. The latter could suggest that the times under GLC limitation/exhaustion could signal some E.coli cells into high stress/lower division rate phenotypic states. Conversely, for S.cerevisiae, the population presents a broader range of sizes across the FSC-A channel initially, decreasing in size and dispersion across time, concentrating the population on lower values. The smaller cell sizes suggest cells spend less time in the G1 phase in agreement to consuming a slower growth metabolite $(\mathrm{ETH})$ [47, 48]. The smaller size could reduce the apparent dilution rate of enzymes and regulatory signals, while higher division times could introduce metabolic heterogeneity 48. The models presented in this work are unsegregated. However, simulations showed higher relative enzyme concentrations and a more heterogeneous distribution of the metabolic states in the mentioned conditions. These characteristics could then help S.cerevisiae to have better fitness across changing environments.

Fig. $7 \mathrm{~B}$ shows that the intermittent feeding profiles generate an oscillating pattern on the relative abundance profiles of the co-cultured microorganisms. The frequency of the population oscillations agrees with the feeding increments, and the amplitude of these oscillations seems to be in inverse relationship with the frequency. A more considerable dispersion for the hi-freq experiment after 30 hours (on the first oscillations after initial GLC exhaustion) is observed. However, this dispersion reduces as the fermenter is operated in this frequency regime achieving similar values to the other two experiment regimes at $80 \mathrm{~h}$. At this time frame, an increase in the population fraction of S.cerevisiae can be observed for both intermittent experiments (Fig. 7). The mean fractional value after 40 -h for S.cerevisiae fraction has values of $\approx 7.14 \%, 15.59 \%$, and $18.35 \%$ for the continuous, low-freq and hi-freq experiments, respectively. These mean values represent an increase for the S.cerevisiae the relative abundance of 2.18 and 2.57 times. Interestingly, the OD600 values across time and its behaviour profiles are relatively similar for the three experimental feeding profiles, achieving between 12 and 14 OD from 30 hours to end of fermentation (S1 File). The latter suggests that the observed changes in relative abundance do not derive from biomass loss but on differential substrate utilisation and fate. It is essential to note that the model presents a significantly smaller oscillation amplitude on the fraction of population than what is observed, causing the predicted value to stay near the maximal fractions observed experimentally but farther to the mean observed values (S1 File). This difference suggests that the cells in the co-culture are even more responsive than expected by the model. The latter is probably because the model is an initial prediction of the behaviour of the co-culture by coupling the macroscopic behaviour of the microorganisms measured and characterised in axenic cultures, without introducing at first changes due to microbial social interactions such as commensalism,
346 

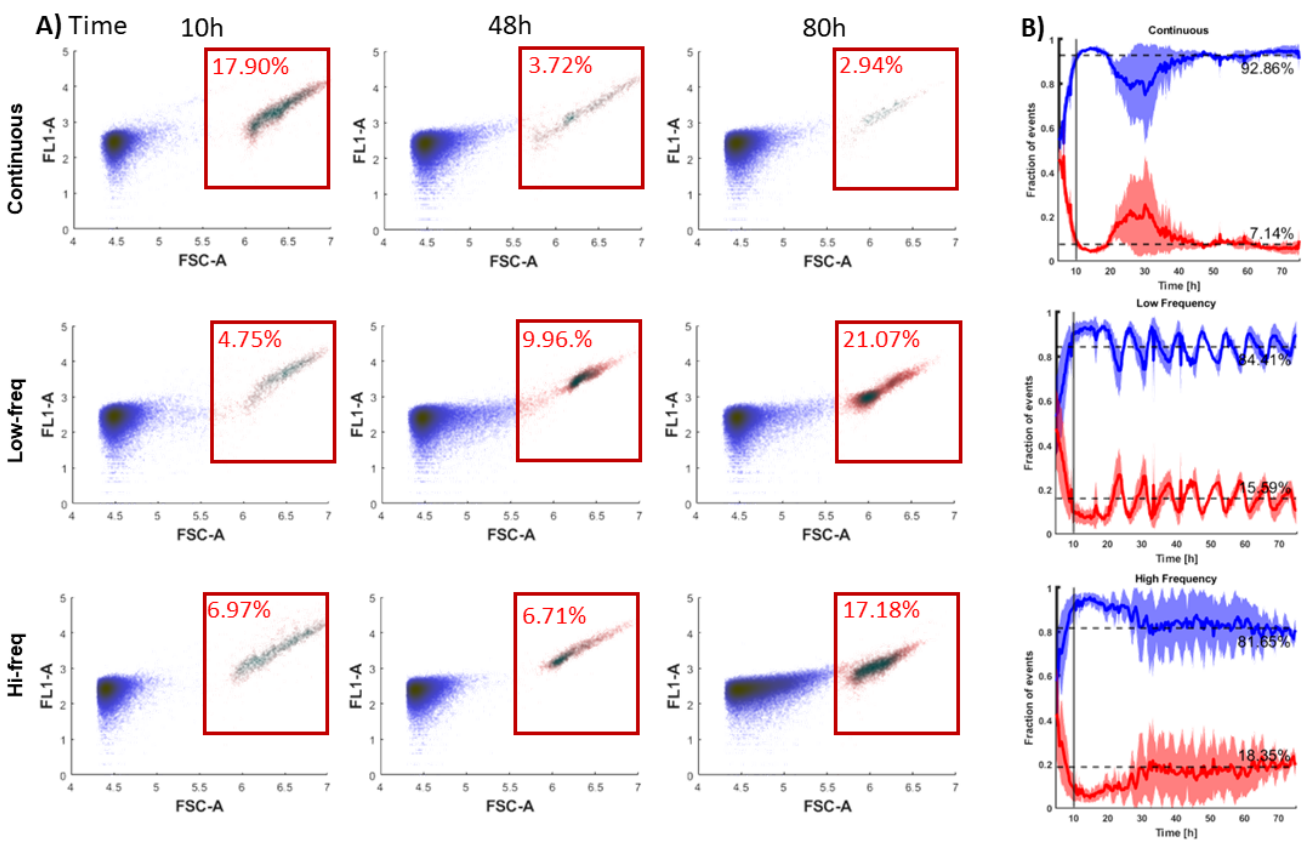

Fig 7. A) FL1-A vs FSC-A FC plots for the semi-continuous fermentations at different pulsating profiles at times 10 (feed start time), 48 and 80 hours. The E.coli centroid is in blue, the S.cerevisiae centroid in red, and with marked gate with percentage of S.cerevisiae B) E.coli (blue) and S.cerevisiae (red) fractional events $/ \mu \mathrm{L}$ vs. time calculations obtained from the online FC data for all feeding profiles.

predatory behaviour, or symbiosis, among others, that could significantly impact strain population sensibility and stability on the system. Nevertheless, the model seems to help find general trends for the competition of the different microorganisms during different intermittent feeding profiles with different dynamics for substrate availability. Probably the most interesting point is the systemic property of the system to enter a state of dynamic stability, in which both strains achieve quasi-stable proportions and concentrations.

In Fig. $8 \mathrm{~A}$ and $\mathrm{C}$, the FSC-A distribution of FC events across the sampling time at 80h for the continuous and Hi frequency experiments are presented. It can be observed that we can find a randomised sampling between both strains in both cases for the entire measurement time. Moreover, the partition between both clustered systems seems to allow small error in segregating events into the two microorganisms categories. The size and apparition of events of different sizes on the sample time are homogeneous. Data suggest that there is not only an enrichment from the intermittent experiment for the S.cerevisiae strain, but also a reduction into the apparent size of the events, as a far less dispersed and lower upper limit on FSC-A can be found for this experimental condition. The sample micrographies comparison for the continuous and intermittent experiments can be found in Fig. 8 B and D, respectively. On the micrography, it can be observed that S.cerevisiae cells are surrounded by attached E.coli, conversely, for the intermittent experiment, this effect is not observed, and almost all observed cells were single cells with no aggregation. Therefore the cells and the Continuous culture would seem more prominent than sole S.cerevisiae cells and would have a wider size distribution given the degree of aggregation, while on intermittent experiments, the absence of this effect would compress the size distribution into a relatively smaller mean size. It is relevant 

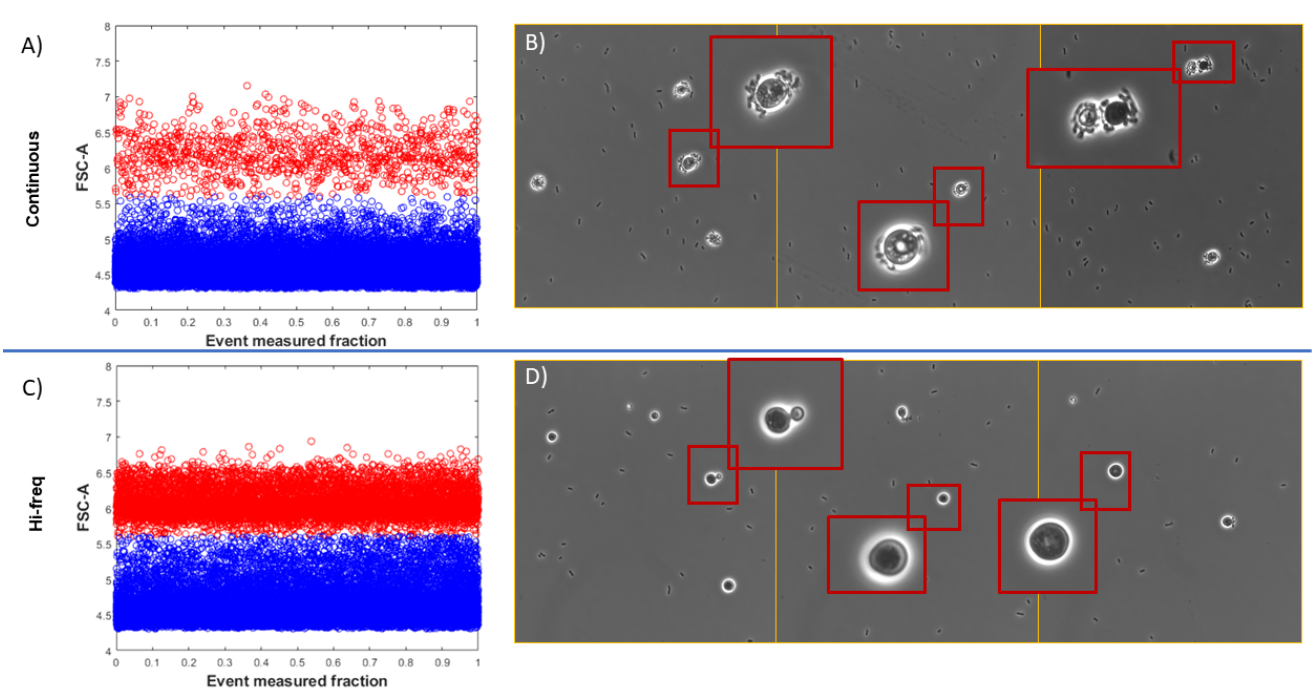

Fig 8. A) and B) Continuous cultivation FSC-A vs event measured fraction plot and micrography samples, respectively, at time 80 h. E.coli (blue) and S.cerevisiae (red). C) and D) Hi-frequency pulsed profile cultivation FSC-A vs SSC-A FC plot and micrography samples, respectively, at time $80 \mathrm{~h}$. On all micrographies, example marked cells have been magnified.

to notice that the intermittent feeding profiles exert more than metabolic changes into the individual cells but also seem to change the microorganism's morphology and social behaviour, probably impacting co-culture dynamics, and strain fixation to a great extent [34]. The latter, in turn, could impact their fitness and survival in a fermentation process by coupling or decoupling specific metabolic profiles and functions and some microbial behaviour profiles and their interactions.

\section{Conclusion}

In this work, we have addressed the possibility of modifying population stability during continuous and semi-continuous co-cultures of yeast and bacteria. We utilised, for this reason, a microorganism pair that, in normal conditions, is expected to have its slowestgrowing or less-fit species washed out during the operation of a continuous reactor with a single carbon source. As presented and discussed in previous sections, we characterised the phenotypic state and metabolic outputs from each particular microorganism in axenic cultures with GLC as the sole carbon source with the aid of a cybernetic modelling approach. Models agreed with known metabolic characteristics of the strains and helped to infer other cellular systemic characteristics such as metabolism regulation and elasticity The E.coli strain presented a highly dominant GLC metabolism which exerted severe constraints on the allocation of cellular resources to other metabolic possibilities, which highly neglected substrate co-utilisation and hinted to more significant times of phenotypic transitions after GLC exhaustion. Conversely, S.cerevisiae presented less marked constraints into the allocation of resources to alternative substrates consumption, allowing higher concentrations of the relative enzymes needed for each substrate catabolism, resulting in a mixed metabolism phenotype and hinting at a more elastic metabolism towards changing environments. The latter two observations derived the hypothesis that intermittent changes in substrate availability could, in specific frequencies, reduce initial 
fitness and growth rate imbalance between the strains. Therefore, we proposed that given these temporal fitness balance changes, dynamic feeding profiles could control population composition, stabilising the unfavored population across fermentation time. With this objective, we constructed a co-culture model based on a virtual strain that contained the metabolic functions of both strains and with compartmentalised biomasses and growth metabolic objectives. The information of the single models, along with a parameter value refinement given by continuous reactor co-culture data, allowed us to profile the potential behaviour of the strains and some of the characteristics of their metabolic interaction. The construction of this virtual co-culture cell model was then used to perform several simulations designed to address the potential population behaviour during various intermittent feeding profiles that brought to light the possibility of increasing the S.cerevisiae population and stability during specific feeding frequencies, continuous step times, and dilution rates. We, therefore, used two different intermittent feeding profiles to be tested experimentally in $1 \mathrm{~L}$ bioreactors. This resulted in oscillating biomass relative abundance profiles with increased mean proportions to 2.18 and 2.57 times their original values for S.cerevisiae.

The oscillations in the relative abundance were found to be achieved without significant changes in total biomass concentration. It was suggested that they arise from a differential substrate utilisation and temporal changes on the strain fitness balance, which signify that S.cerevisiae can temporarily grow faster than E.coli, allowing its stabilisation in the co-culture. Furthermore, we found that the intermittent profiles not only have impacts on the substrate utilisation and fate profiles but also on microorganism social behaviour and interaction as we found that E.coli is surrounding S.cerevisiae cells in fully continuous cultures. By contrast, they do not significantly form cell clusters during intermittent cultures. Given the additional changes observed in intermittent conditions, model simulations tended to overestimate the final mean concentrations of the latter mentioned strain. This is due to its simplified profile, accounting only for the extracellular metabolite interactions and competitions; however, it was enough to render the general trends on the metabolic behaviour towards the fitness and stabilisation increases of S.cerevisiae. It is relevant to state that the model can be extended within the same framework to address more complex metabolite functions, internal fluxes, specific inhibitions, and interactions using the same cybernetic modelling approach. Therefore, the presented framework could be extended and refined with further experimental data that account for different characteristics of specific microbial interactions. The model presented represents the first step in constructing a modular framework and virtual co-culture strains database to understand and control microorganism populations during continuous bioprocesses.

\section{Supporting information}

S1 File. Simulation Supporting information. File containing Willmott index values for the simulation comparison to data, response surfaces for Dilution rate, frequency and step feed time simulation solution space, Behavior curves for relative enzyme variable for all simulations, and experimental data and model simulations for the high and low frequency intermittent feeding regimes.

\section{Acknowledgments}

We would like to thank the Era-Cobiotech established based on the H2020 European framework for providing funding (Contibio project). Wallonia is also gratefully ac- 
knowledged for financial support. JAM is supported by a post-doctoral grant (Contibio project). FM is supported by a post-doctoral grant (Sunup project, supported by Wallonia). LH is supported by a FRIA PhD grant provided by the Belgian Fund for Scientific Research (FNRS). MD is supported by a $\mathrm{PhD}$ grant provided by the Belgian Fund for Scientific Research (FNRS) in the context of an Era-Net Aquatic Pollutant project (ARENA). We would also like to thank Vincent Vandenbroucke for its help reviewing this manuscript.

\section{References}

1. Lawson CE, Harcombe WR, Hatzenpichler R, Lindemann SR, Löffler FE, O'Malley MA, et al. Common principles and best practices for engineering microbiomes. Nature Reviews Microbiology. 2019;17(12):725-741. doi:10.1038/s41579-019-02559.

2. Binder D, Drepper T, Jaeger KE, Delvigne F, Wiechert W, Kohlheyer D, et al. Homogenizing bacterial cell factories: Analysis and engineering of phenotypic heterogeneity. Metabolic Engineering. 2017;42(March):145-156. doi:10.1016/j.ymben.2017.06.009.

3. Losoi PS, Santala VP, Santala SM. Enhanced Population Control in a Synthetic Bacterial Consortium by Interconnected Carbon Cross-Feeding. ACS Synthetic Biology. 2019;8(12):2642-2650. doi:10.1021/acssynbio.9b00316.

4. Escalante A, Elena Rodríguez M, Martínez A, López-Munguía A, Bolívar F, Gosset G. Characterization of bacterial diversity in Pulque, a traditional Mexican alcoholic fermented beverage, as determined by $16 \mathrm{~S}$ rDNA analysis. FEMS Microbiology Letters. 2004;doi:https://doi.org/10.1016/j.femsle.2004.04.045.

5. Wang L, York SW, Ingram Lo, Shanmugam KT. Simultaneous fermentation of biomass-derived sugars to ethanol by a co-culture of an engineered Escherichia coli and Saccharomyces cerevisiae. Bioresource Technology. 2019;(273):269-276. doi:https://doi.org/10.1016/j.biortech.2018.11.016.

6. Ly S, Bajoul Kakahi F, Mith H, Phat C, Fifani B, Kenne T, et al. Engineering Synthetic Microbial Communities through a Selective Biofilm Cultivation Device for the Production of Fermented Beverages. Microorganisms. 2019;7(7):206. doi:https://doi.org/10.3390/microorganisms7070206.

7. Delvigne F, Baert J, Sassi H, Fickers P, Grünberger A, Dusny C. Taking control over microbial populations: Current approaches for exploiting biological noise in bioprocesses. Biotechnology Journal. 2017;12(7). doi:https://doi.org/10.1002/biot.201600549.

8. Macuso CP, Lee H, Abreu C, Gore J, Khalil AS. Environmental fluctuations reshape an unexpected diversity-disturbance relationship in a microbial community. eLife. 2021;10:1-31. doi:https://doi.org/10.7554/eLife.67175.

9. Chen Y, Kim JK, Hirning AJ, Josić K, Bennett MR. Emergent genetic oscillations in a synthetic microbial consortium. Science. 2015;349(6251):986-989. doi:10.1126/science.aaa3794.

10. Kussell E, Leibler S. Phenotypic Diversity, Population Growth, and Information in Fluctuating Environments. Science. 2005;309(5743):2075-2078. doi:10.1126/science.1114383. 
11. Thattai M, van Oudenaarden A. Stochastic Gene Expression in Fluctuating Environments. Genetics Society of America. 2004;(167):523-530.

12. Jaruszwicz J, Kimmel M, Lipniacki T. Stability of bacterial toggle switches is enhanced by cell cycle lenghtening by several orders of magnitude. Physiocal Review E. 2014;(89):26. doi:dx.doi.org/10.1103/PhysRevE.89.022710.

13. Lendenmann U, Mario S, Thomas E. Growth kinetics of Escherichia coli with galactose and several other sugars in carbon-limited chemostat culture. Can J Microbiol. 2000;(46):72-80.

14. Groh A, Krebs J, Wagner M. Efficient solution of an inverse problem in cell population dynamics. Inverse Problems. 2011;27(6). doi:10.1088/0266-5611/27/6/065009.

15. Ramkrishna D, Song HS. Dynamic Models of Metabolism: Review of the Cybernetic Approach. Bioengineering,Food, and Natural Products. 2012;58(4):986-997. doi:10.1002/aic.

16. Kompala DS, Ramkrishna D, Jansen NB, Tsao GT. Investigation of bacterial growth on mixed substrates: Experimental evaluation of cybernetic models. Biotechnology and Bioengineering. 1986;28(7):1044-1055. doi:https://doi.org/10.1002/bit.260280715.

17. Martinez JA, Bulte DB, Contreras MA, Palomares LA, Ramirez OT. Dynamic Modeling of CHO cell Metabolism Using the Hybrid Cybernetic Approach With a Novel Elementary Mode Analysis Strategy. Frontiers in Bioengineering and Biotechnology. 2020;(8):279.

18. Varner J, Ramkrishna D. Metabolic Engineering from a Cybernetic Perspective Aspartate Familiy of Amino Acids. Metabolic engineering. 1999;1(MT980104):88116. doi:1096-7176/99.

19. Song HS, Ramkrishna D, Pinchuk GE, Beliaev AS, Konopka AE, Fredrickson JK. Dynamic modeling of aerobic growth of Shewanella oneidensis. Predicting triauxic growth, flux distributions, and energy requirement for growth. Metabolic Engineering. 2013;15:25-33. doi:https://doi.org/10.1016/j.ymben.2012.08.004.

20. Aboulmouna L, Raja R, Khanum S, Gupta S, Maurya MR, Grama A, et al. Cybernetic modeling of biological processes in mammalian systems. Current Opinion in Chemical Engineering. 2020;30:120-127. doi:https://doi.org/10.1016/j.coche.2020.100660.

21. Ramkrishna D, Song HS. Analysis of Bioprocesses. Dynamic Modeling is a Must. materialstoday:proceedings. 2016;3:3587-3599.

22. Willmott CJ. Some Comments on the Evaluation of Model Performance. Bulletin of the American Meteorological Society. 1982;63(11):1309 - 1313. doi:10.1175/15200477(1982)063;1309:SCOTEO ¿2.0.CO;2.

23. Willmott CJ. ON THE VALIDATION OF MODELS. Physical Geography. 1981;2(2):184-194. doi:10.1080/02723646.1981.10642213.

24. Ramos Pereira H, Meschiatti tOjAyvndhp Monica Cristina and Celia de Matos Pires, Regina and Constatino Blain, Gabriel;.

25. Verduyn C, Postma E, Scheffers WA, Van Dijken JP. Effect of benzoic acid on metabolic fluxes in yeasts: A continuous-culture study on the regulation of respiration and alcoholic fermentation. Yeast. 1992;8(7):501-517. doi:https://doi.org/10.1002/yea.320080703. 
26. Haddad SA, Lindegren CC. A Method for Determining the Weight of an Individual Yeast Cell. Applied Microbiology. 1953;1(3):153-156. doi:10.1128/am.1.3.153156.1953.

27. Sauer U, Lasko DR, Fiaux J, Hochuli M, Glaser R, Szyperski T, et al. Metabolic Flux Ratio Analysis of Genetic and Environmental Modulations of ¡i_Escherichia colij/ii Central Carbon Metabolism. Journal of Bacteriology. 1999;181(21):66796688. doi:10.1128/JB.181.21.6679-6688.1999.

28. Sohn SB, Kim TY, Lee SY. Genome-scale metabolic model of the fission yeast schizosaccharomyces pombe and the reconciliation of insilico/in vivo mutant growth. BMC Systems Biology;16, number $=49$, pages $=1-12$, year $=2012$, doi $=$ https://doi.org/10.1186/1752-0509-6-49.

29. Nguyen TM, Telek S, Zicler A, Martinez JA, Zacchetti B, Kopp J, et al. Reducing phenotypic instabilities of a microbial population during continuous cultivation based on cell switching dynamics. Biotechnology and Bioengineering. 2021;118(10):3847-3859. doi:https://doi.org/10.1002/bit.27860.

30. Fragoso-Jimenez J, Baert J, Nguyen T, Liu W, Sassi H, Goormaghtigh F, et al. Growth-dependent recombinant product formation kinetics can be reproduced through engineering of glucose transport and is prone to phenotypic heterogeneity. Microbial cell Factories. 2019;26:18-26. doi:https://doi.org/10.1186/s12934-0191073-5.

31. Diderich JA, Schepper M, van Hoek P, Luttik MAH, van Dijken JP, Pronk JT, et al. Glucose Uptake Kinetics and Transcription of HXTGenes in Chemostat Cultures of Saccharomyces cerevisiae*. Journal of Biological Chemistry. 1999;274(22):1535015359. doi:https://doi.org/10.1074/jbc.274.22.15350.

32. Förster AH, Gescher J. Metabolic Engineering of Escherichia coli for Production of Mixed-Acid Fermentation End Products. Frontiers in Bioengineering and Biotechnology. 2014;2. doi:10.3389/fbioe.2014.00016.

33. Jawed K, Yazdani SS, Koffas MA. Advances in the development and application of microbial consortia for metabolic engineering. Metabolic Engineering Communications. 2019;9:e00095. doi:https://doi.org/10.1016/j.mec.2019.e00095.

34. Winkle JJ, Karamched BR, Bennett MR, Ott W, Josić K. Emergent spatiotemporal population dynamics with cell-length control of synthetic microbial consortia. PLOS Computational Biology. 2021;17(9):1-23. doi:10.1371/journal.pcbi.1009381.

35. Namjoshi AA, Ramkrishna D. Multiplicity and stability of steady states in continuous bioreactors: Dissection of cybernetic models. Chemical Engineering Science. 2001;56(19):5593-5607. doi:10.1016/S0009-2509(01)00166-X.

36. Delvigne F, Goffin P. Microbial heterogeneity affects bioprocess robustness: Dynamic single-cell analysis contributes to understanding of microbial populations. Biotechnology Journal. 2014;9(1):61-72. doi:https://doi.org/10.1002/biot.201300119.

37. Sassi H, Nguyen TM, Telek S, Gosset G, Grünberger A, Delvigne F. Segregostat: a novel concept to control phenotypic diversification dynamics on the example of Gram-negative bacteria. Microbial Biotechnology. 2019;12(5):1064-1075. doi:https://doi.org/10.1111/1751-7915.13442. 
38. Stephens K, Pozo M, Tsao CY, Hauk P, Bentley WE. Bacterial coculture with cell signaling translator and growth controller modules for autonomously regulated culture composition. Nature Communications. 2019;10:4129. doi:https://doi.org/10.1038/s41467-019-12027-6.

39. Bader J, Mast-Gerlach E, Popović MK, Bajpai R, Stahl U. Relevance of microbial coculture fermentations in biotechnology. Journal of Applied Microbiology. 2010;109(2):371-387. doi:https://doi.org/10.1111/j.1365-2672.2009.04659.x.

40. Rodríguez-Verdugo A, Vulin C, Ackermann M. The rate of environmental fluctuations shapes ecological dynamics in a two-species microbial system. Ecology Letters. 2019;22(5):838-846. doi:https://doi.org/10.1111/ele.13241.

41. Abreu CI, Andersen Woltz VL, Friedman J, Gore J. Microbial communities display alternative stable states in a fluctuating environment. PLOS Computational Biology. 2020;16(5):1-17. doi:https://doi.org/10.1371/journal.pcbi.1007934.

42. Klitgord N, Segrè D. Environments that Induce Synthetic Microbial Ecosystems. PLOS Computational Biology. 2010;6(11):1-17. doi:https://doi.org/10.1371/journal.pcbi.1001002.

43. Dolinsek J, Goldschmidt F, Johnson DR. Synthetic microbial ecology and the dynamic interplay between microbial genotypes. FEMS Microbiology Reviews. 2016;40(6):961-979. doi:https://doi.org/10.1093/femsre/fuw024.

44. Mee MT, Wang HH. Engineering ecosystems and synthetic ecologies. Mol BioSyst. 2012";8:2470-2483. doi:https://doi.org/10.1039/C2MB25133G.

45. Tan C, Reza F, You L. Noise-Limited Frequency Signal Transmission in Gene Circuits. Biophysical Journal. 2007;93(11):3753-3761. doi:https://doi.org/10.1529/biophysj.107.110403.

46. Tostevin F, ten Wolde PR. Mutual Information between Input and Output Trajectories of Biochemical Networks. Phys Rev Lett. 2009;102:218101. doi:10.1103/PhysRevLett.102.218101.

47. Johnston GC, Ehrhardt CW, Lorincz A, Carter BL. Regulation of cell size in the yeast Saccharomyces cerevisiae. Journal of Bacteriology. 1979;137(1):1-5. doi:10.1128/jb.137.1.1-5.1979.

48. O'Brien EL, Van Itallie E, Bennett MR. Modeling synthetic gene oscillators. Mathematical Biosciences. 2012;236(1):1-15. doi:https://doi.org/10.1016/j.mbs.2012.01.001. 